\title{
Communication of the "Objective Reality" as Signal to the Senses in Orchestrated Non-Reductive way
}

\author{
AK Mukhopadhyay* \\ Professor of Pathology, North DMC Medical College, India
}

*Corresponding author: AK Mukhopadhyay, Professor of Pathology, North DMC Medical College, Delhi, India.
Received Date: June 02, 2019

Published Date: July 16, 2019

\begin{abstract}
Consciousness cannot be articulated. In the unconditional state it is inexpressible. However, this Transcendental Reality could be naturalized internally by the human brain, which has been evolutionarily primed to do this. On the other side, externally, this has been a spontaneous happening in nature. Two pathways meet, although over a long time gap, at the sensory apparatus as "perception" of the experience of the Objective Reality (OR). This paper describes the steps in this process with involvement of four primary operations. From ontological point of view the author identifies the operators as consciousness, self, life, mind and information working together with the brain and shows that they form an orchestra which has been traditionally called psyche, which acts as inter-phase between brain-bound and brain-independent consciousness. From epistemological point of view the operations, labeled bottom up as operation I, II, III and IV which although occur in both inside the brain and in the external world, get more emphasis in the description of happenings in the external world. They seem to form a systems of orchestrated communication of the Objective Reality in a non-reductive way to the senses as signal. In this communication, Consciousness remains the First, Mind and its operation is the Final common path, and the Signal signifies the closure of causal drainage at the level of the matter..
\end{abstract}

Keywords: Brain-independent consciousness; Objective Reality; Psyche as inter-phase; Cognitive orchestra; Layers in between patterns of neuronal signal and consciousness; Working definitions of consciousness; Attitude; ZPE state of Brain

\section{Introduction}

American Philosopher John Searle has made a significant statement, "consciousness is a philosophical problem, but it will have scientific solution". What is this solution? Is it development of a fool-proof theory of consciousness? Or, consciousness fully explained! Unlikely! Consciousness cannot be approached or explained by or confined to any Theory. It does not matter how much of rigor from intelligence the theory upholds! Consciousness is intellectually incomprehensible, probably inexpressible and not fully describable in mathematical, verbal or non-verbal language! This property has created all clouds about its nature! However, consciousness is not hotchpotch phenomenon of the psyche, nor it could be ignored as blah, blah within the brain! Consciousness could be made intelligible and sensible at the level of Boolean logic by descending the steps through inferential logic, formal logic and fuzzy logic.

At present there are several competing theories on this consciousness, such as Global Workspace Theory of Bernard Baars [1], Integrated Information Theory of G. Tononi [2], Phenomenal and
Access Consciousness Theory of Ned Block [3], and Orchestrated Objective Reduction Theory of Hameroff and Penrose [4], besides several other Representation theories including First Order-, and Higher Order-Representation Theories! All of these theories are in the context of the functions of the brain as if the brain is the source of consciousness! Consciousness is generated "somehow" within the brain from the pattern and dynamicity of neural signalling! And, the rescuer for this "somehow" for most of them is quantum physics, quantum events at the sub-cellular structures of neurons [5], or physics [6], physical phenomena in the external world.

My position, in this respect, is different and is also clear [7]. The brain can neither generate, nor can use consciousness. It is consciousness which uses brain for its manifestations! The author is not alone in upholding this idea that the brain is not the source of consciousness! There is radio reception theory of Henri Bergson regarding brain consciousness relationship, extracerebral consciousness of John Eccles, television analogy for brain function of George Wald. According to Aldous Huxley, the brain is a 
biological reducing valve! Sir William James, the Father of American psychology, has the transmissive theory of brain function regarding consciousness. Even the Hippocrates, the Father of modern medicine holds such view!

Physics of "life", physics of "self", physics of information and quantum physics are intellectual manifestations of consciousness. External world is manifestation of cosmic consciousness, so also we and our organs including the brain. Then, what could be the way to the Searle's solution? The way to the solution is perhaps based on the fact that consciousness is not merely an ontological entity. Consciousness has operations at the abstract level, which could be logically connected with operations familiar in physical, informational, phenomenal nature and in nature of "life". The way to the solution is to hold consciousness and its operations as inviolable constants and treating all other constants, natural or man-made, as flux within our cognitive frame.

This paper works on how the Objective Reality (OR) of Unconditional Consciousness is communicated through potent causal agents as signal to senses in an orchestrated, but nonreductive way! In this paper, we hold consciousness as the First, mind and its operation the final common path, and the signal in the material plane as observable result of this communication. The matter remains the domain where the causal drainage is closed.

\section{The Objective Reality (OR)}

What is this OR? The objective reality is the reality that exists independent of me, you and us. Every other person, in principle, is able to verify every aspect of the objective reality. Anything that cannot be verified in this way could not be the objective reality. In the context of the science for consciousness, the OR is the unconditional and ground consciousness supporting the fabrics of both external and internal reality, which could be 'sensed' as Quality. May be in religious jargon, this is sort of an "External God" and in philosophical language this has been described as the "Ideal". Consciousness as $\mathrm{OR}$ is recognized in science as the most hard issue for any business transaction not only in neuroscience and anthropology, but also in the economics, industry (neuro-marketing, user interface in designing) and even in military establishment (information war, lie detector, mind-reading in spying etc.) [8]. Richard Thaler has already won the Noble Prize in 2017 on behavioral economics [9].

The attributes of the OR could be stated as universal wisdom, 'will', the decision of the systems universe or the systems of multiple universe(s)! Always, there is a transcendental element in all such attributes. The attributes transcend individual (as in Transpersonal psychology), collective (as in social psychology), the universe (the cosmology) and often multiple universe(s)! None of such attributes of OR could be perceived by senses directly. This requires, what in science jargon is called, "reduction".

\section{Why "reduction" is necessary and that too in objective way?}

This OR is so huge, overarching and dynamic that it is wise to bring this down from an infinite scale to a measurable scale to make it intelligible and sensible. The goal is to understand, and to make other understand how the scheme in the nature brings the quality at the highest level down as the perception by senses at the bottom level. Why it is so necessary? In the quest for consciousness by human being, this "reduction" seems necessary for understanding the design of nature and consciousness, to express the design from first person's perspective in third person's perspective and for carrying out the instruction communicated through its signature signal. In the external nature, this "reduction" is necessary for its manifestation with incredible diversity. One of the diversities is creation of "receptors" for its signature signal in the selected and primed living entity! We are also hoping that the external pathway might unveil the mechanism of harnessing the inexhaustible source field of unconventional dark energy for public utility.

\section{What OR is eventually "reduced" to, and what could "reduce" this OR?}

The OR is "reduced" to different signals in nature which are detectable, measurable and verifiable. In the internal route, what this objective reality is ultimately reduced to is "perception", which could be correlated with the pattern and dynamicity of the signal frequency of a live cell or signal oscillation in the specific or global neuronal assembly, which could be observed by live cell technology or neuro-imaging techniques!

In the external world such natural processes have been happening or occurring spontaneously since the time of generation of multiple universe(s)! At the present stage, any scenario beyond multiple universe(s) seems intellectually incomprehensible! This is our cognitive limit! Multiple universe(s) cascade down to our universe, the galaxies, the stars, to our planet! There is information loss at three voids; inter-universal, inter-galactic and interstellar void. One thing, therefore, is to be remembered that all signals as seen in the sensory world are not the signature signal of the OR of the Multiversity! Many signals generated at the zero-point energy state are meant for creation and maintenance of nature's incredible diversity. So also signal systems from the deepest level exist for creation of specific "receptor", which once developed, could be useful in selected and primed living entity for understanding this OR itself. Therefore, an evolved human being having such receptor, could also engage in such communication by using his brain! There is a role of human psyche and the instrument brain in such communication of the OR as internal perception of the signal.

\section{What is the Nature of this "Reduction"?}

Is this really a process of "reduction"? Or, something else! This reduction is surely not breaking the whole into its parts! In the subjective wing we use the terms as realization, transformation, representation and finally communication. In outside worldly pathway, this could be best described as holonomic continuation of the pattern and the dynamicity of the whole, and subsequently holographic representation of the pattern of the whole (as observed in form of fractals in inanimate and animate objects, in the flora and fauna of nature) before it is finally communicated as signal/pixel in the material plane. Astonishing aspect of this process is that there is 
substance/category differentiation in every step. In spite of this, the "reduced" ones could retain the quality of the whole. The process as a whole could be best described as a manifestation of the whole as a cognitive organ for communication.

\section{How addressing of this issue began?}

Considering the issue, the present author coined the term and the Idea of supracortical consciousness in 1985 [10] and has been developing the idea further through publication [11] of books and papers since then. According to this Idea, animal neuroaxis operates like an inverted tree with roots open in the eternity and branches below down as peripheral nerves. The very concept of supracortical consciousness brings a completely new Worldview, since the "Power" is no longer with the brain but is with consciousness. The idea is revolutionary as well! It is revolutionary because it absolves (and then transforms) a large number of man-made divides such as subjective-objective divide, evolution-creation divide etc., at macro scale, and left-brain right-brain divide, intellectual-emotional brain divide, thinking-feeling brain divide etc., at the scale of the brain.

Operation of Supracortical consciousness rejuvenates, rediscovers, redefines and reinvents the self and functions of the brain. The present author in the chapter eight of his book, The Millennium Bridge [12], propounds the long term consequences of such supracortical awareness, manifested on the ground as the development of a new brain, which has been called the "Brain" of a brain, over the top of the cerebral cortex, but regionalized in bi-hemispherical location, and formed out of new recruitment of cells from the stem cell pool (Figure 1) around the periventricular region of "brain marrow" [13]! This is the answer to an enquiry on development of the required infrastructure at the structural level for an inter-phase between brain-bound and brain-independent consciousness! (Figure 1).

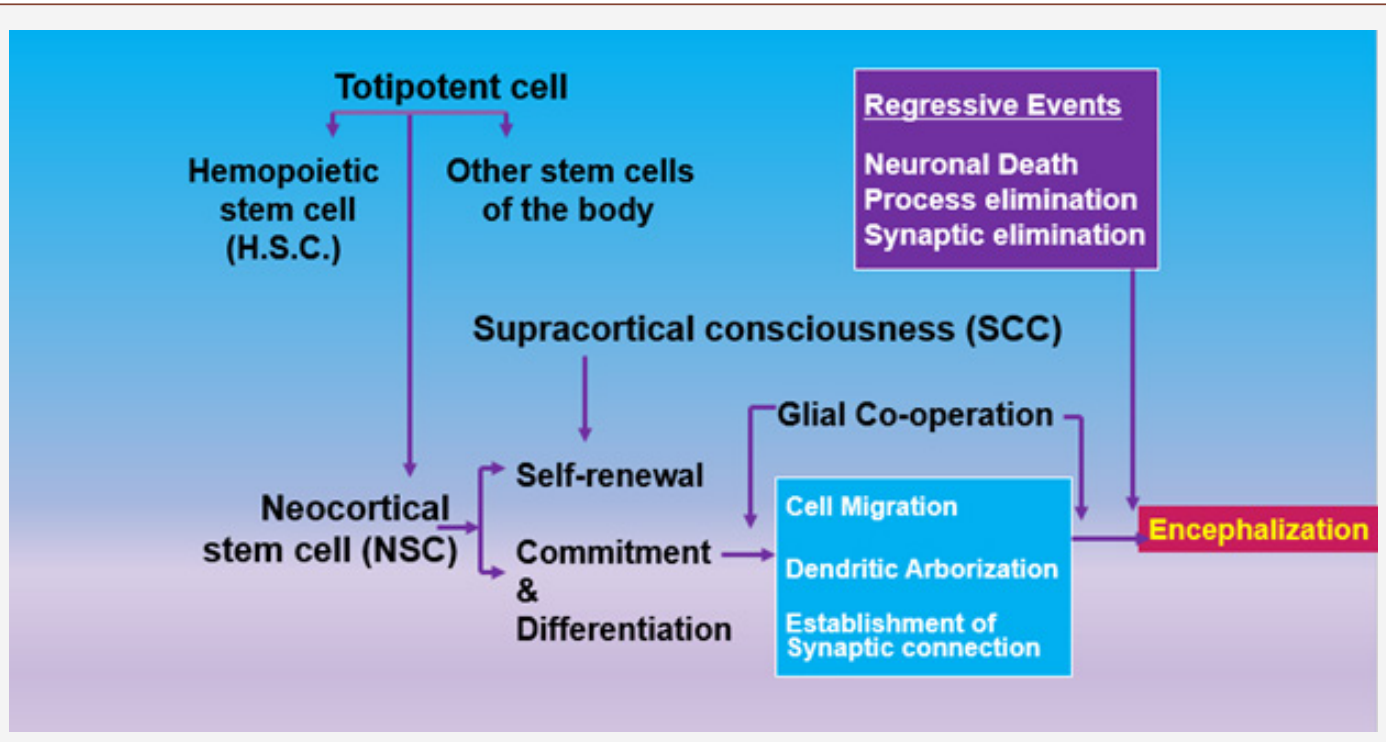

Figure 1: This schematic diagram shows how the awareness of supracortical consciousness could stimulate stem cells for renewal in the brain for reorganization of neuronal network through progressive and regressive events that are eventually involved in encephalization. The Figure was first published in Author's book, The Dynamic web of Supracortical Consciousness, in 1987.

\section{Theory of Downward Causation in Philosophy}

Theory of downward causation as presently exists in philosophy is marred with several issues such as issue of causality (causal closure, causal exclusion, property dualism and supervenience and causal drainage etc.), issue of intentionality, issue of presence or absence of an agent which cognizes, phenomenological issue, and what takes the final call in the communication process! Philosopher Kim's arguments [14], although not the subject of the paper, are relevant here for the interested readers. The downward causation theory, however, could not come out with a solution because of the lack of clarity on the inter-phasing systems. The 'Mind' is one and all for the theory. Some theory of mental causation, although, admits consciousness but is clueless how consciousness is related to mind and what is the exact relationship of the duo! The fact that mind is a property of a living entity has not been accounted in this theory. The relationship between 'life' and 'mind' has not been explored! The question whether this mind has any reporting authority remains unattended. Mind reports to what? Common sense says we control our mind. The 'self' controls the mind. Mind, in the language of management, reports to self. There is an element of self-organization in this causal process. This point has not been accounted in the theory of downward causation. Besides, the versatile role of Information as a non-observable causal entity, as a currency of business transaction in the entire process has not been looked into the existing downward causation theory!

The issue of how consciousness becomes the physical universe has been attempted in an interesting paper from Menas Kafatos, Rudy Tanzy and Deepak Chopra [15]. In the recent past, the neuroscientist Karl Pribram had the idea that the brain is an interfacing organ having a personal universe which communicates with the real outside universe [16]. Beichler [6] in his two-way approach to consciousness research, argues for extracerebral and intracerebral routes to consciousness. Benjamin Libet's experimental evidence [17] also hints towards intracerebralextracerebral communication, although he had not been articulate on this. Kak [18] is of the opinion that the cosmic architecture is 
mirrored in our brain. The question is how? In this paper, we are readying to develop this inter-phase in the following section.

\section{Steps in this Communication}

What are the steps in this communication? Surely, it is not a single step process! Since the "reduction"/communication can be done by an evolved human being and it has also been happening spontaneously in nature, we can separately narrate the steps in the context of human being and in the context of out-worldly nature.

A. Steps in communication through the brain in an evolved human being:

Step I: Objective Reality of the quality is realized as the subjective experience of the design of nature-consciousness.

Step II: The experience of the design is next transformed into the Architecture of the knowledge systems.

Step III: The architecture of knowledge systems is subsequently represented as combinatorial Geometry of several interrelated and relevant pieces of Information.

Step IV: The combinatorial geometry of information is finally communicated as Signal. The signal is energy expressed in arithmetic of space and time (frequency: space per unit time).

\section{B. Steps in the Communication as executed by Nature:}

Step I: The Will/Quality emerging out of operation of consciousness is communicated to operations of 'Life' and 'Self'.

Step II: Next, the operations of 'Life' formulates the purpose of communication.

Step III: The 'Self' organizes phenomenology according to the dictates of the purpose as churned out by life-operation.

Step IV: Information responsible for the phenomenon is communicated by the operation of 'Mind' as signal to the "beable" quantum particle/wave, or to the receptive classical fields such as electromagnetic fields.

Subsequently the communicated information, the signal, results in micro events as observable in quantum physics, or macro events at the level of classical physics.

\section{How the $\mathrm{OR}$ is Communicated? What are the Operations and the Operators?}

This is the issue of all issues, what how and when about the nature's skill of communication from the level of OR to the level of senses. To explore this is the road ahead! At this stage it is assumed that this is being done through a cascade of operations, which are at least four in number. Each of these operations might have subsystem operations. But at this stage we are not trying to dissect those out. Whenever there is an abstract operation, in all possibility there would be an operator!

From operation to operator, there results in a shift of enquiry from epistemological point of view to ontological point of view. However, as we consider that the operators as entity/agent in phase operates in the sub-quantum depth of nature, there happens a fusion of ontology and epistemology. Such distinction between the two becomes next to impossible. In the sub-quantum and subsub-quantum phase of nature, the operators are identified by their operations and the operations are identified with the operator. The term "operator" gets more emphasis for downward causation in the internal world while the term "operation" gets more emphasis during description of causal cascading in the external world.

Earlier, we have described four steps in this communication. There are at least four operations one each in every step. For topdown description, we may label those as operation IV, III, II and I respectively. The process could also be investigated bottom-up as operation I, II, III and IV. The first operation (Operation IV) at the top is realization of OR into subjective experience. Subjective experience is next transformed by operation III to an architecture of knowledge system. For further simplification, the formal knowledge system representing global information is delivered (Operation II) in form of a semantic information. At the bottom level, this informative expression is communicated by operation I as energy expressed sensibly in arithmetic of space and time as signal/pixel: as space per unit time, which is the frequency of a signal. The system fulfils the minimally required operational orchestra for such communication process. Why minimum? Because, there could be operation I-A, B, $C$ etc., and operation II-A, B, C etc., and so on. However, the minimal framework of this orchestra remains unchanged.

In simple terms, the quality/wisdom/worldview is realized as a powerful experience which has a far-reaching organizing effect in life of the subject to begin a new paradigm according to the new Worldview realized and embodied. The contents of the paradigm are composed into doable hypotheses by operation of self. Hypothesis is downloaded from self to mind as interrelated pieces of information. The packed concept within the information is then communicated as signal to senses (Figure 2).

The cascade above could be described in the language of science of information (Figure 3). Wisdom/Worldview occupies minimum "space" with maximum content, crystallized as heavily compressed sphere, which is a distinguished 'point' in void or a 'moment' in that timelessness, which for the sake of description may be called as "information crystal". This Point of wisdom encased as information crystal undergoes an exalted decomposition into information manifold. From a point generates several spheres piled one above the other in unique design as information manifold. From this information manifold gets composed several copies of global information, upholding a spherical geometry of the Gödelian information. This is followed by downloading of Gödelian information within self as semantic information in mind. Semantic information is made by combinatorial geometry of several interrelated and relevant pieces of trifoliate information (measurable folium works with material world, content folium works with mind and intent folium works with self). The final step is communication of this semantic information as a signal/pixel which works with the senses and leads to perception of the quality of the whole (Figure 3). 


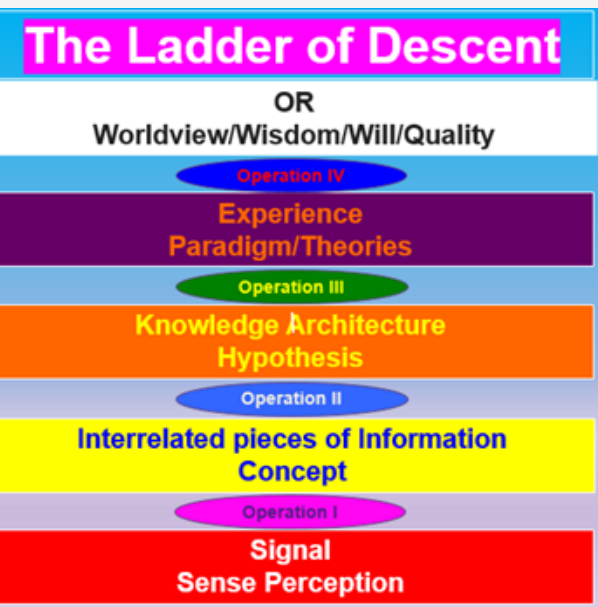

Figure 2: Descending cascade from the OR to the senses. The perception is in the domain of senses. The concept is in the mind. The hypothesis is made by the self. The Theory is build up from experiences in life. The paradigm followed in life is built up from multiple theories related to each other. The Worldview transcends the individual system. The umbilical cord between the Worldview and the experience exists because of the operation of consciousness.

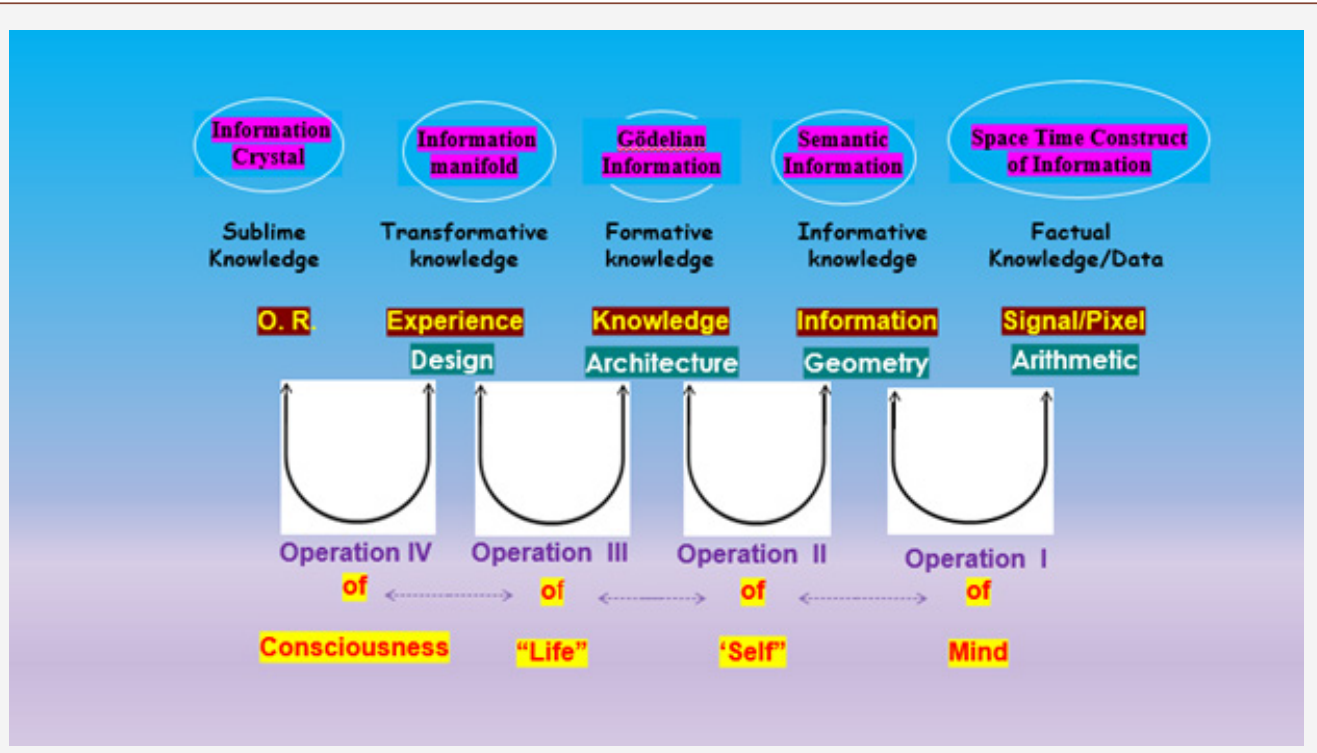

Figure 3: The steps and operations involved in cascading down of crystal information into information manifold, Gödelian information, semantic information and finally to signal/pixel. The figure also shows how OR cascades down as an experience, knowledge, information and signal, how quality cascades down as design, architecture, geometry and arithmetic and how sublime knowledge cascades down to transformative knowledge, formative knowledge, informative knowledge and factual (data-based) knowledge. Four operations and the respective operators by name are shown at the bottom of the figure.

The readers may take a note of an interesting happening during this period. When the "whole" is cascading down the body to be perceived by senses, there has been an accompanying refinement of the senses as well. This happens in order to make the senses capable of interacting with similar signals in the outer world. The circle becomes complete at the level of the senses, at the level of the perception. What has been naturally and spontaneously happening in nature by the downward manifestation of the "whole" as signals, could be perceivable by the senses from the external world too since the senses are primed and ready to perceive the "whole" from signature signal both from inside and outside! At this stage/phase, there is no difference between external and internal perception. God outside and God inside become identical. Quality sensed as perception and quality realized from the inner experience remains identical. The circularity of the process is completed by concurrence of the co-ordinates of operations in the external and internal world.
We are at the doorstep of the discipline of Psychotronics, which is defined by United States Pychotronic Association as, "the science of mind-body-environment relationship, an interdisciplinary science concerned with the interaction of matter, energy and consciousness" [19]. We are also at the reconciliation stage of two approaches to consciousness, external and internal, as elaborated by Beichler [6].

\section{Operators and Operations make an Orchestra}

Let us revise what all members constitute this orchestra? To name them as operator, they are consciousness, life, self, mind and information. The nomenclature of these ontological agents probably has evolved following interpersonal concurrence and consensus arching over the period of cognitive refinement across several civilizations and cultures. Realization of the objective reality into subjective experience requires operation of what we have 
called consciousness (Figure 3). The quality of OR is experienced as "qualia" by operation of consciousness. Transformation of subjective experience into knowledge system requires operation of Life. It is self, which could represent the wholeness of knowledge encapsulated in the subjective experience. We have called that mind, which could reduce this meaningful information into space time combination as space per unit time, which is frequency of a signal or the graphics of a pixel. This has become customary to follow such ontological cascade in describing the inner pathway.

In this inner pathway each of the members operates in their respective phase as powerful causal agent, all are autonomous within the perimeter of holonomy, remaining connected with other's operation to produce systems where the whole determines the subsystems and in the reverse, the subsystems determine the outcome from the whole (Figure 4). In this sense, they form an orchestra. The members of the orchestra maintain a hierarchy (Figure 4). Consciousness is at the Top. Next down in line are 'self' and 'life' (sub-sub-quantum nest, nest IV of nature). Between themselves, 'life' and 'self' maintain a tangled hierarchy, a hierarchy as observed between proverbial yang and yin! Next down the line, are operators such as 'mind' and information along with memory, intelligence and emotion (sub-quantum nest, nest III of nature).

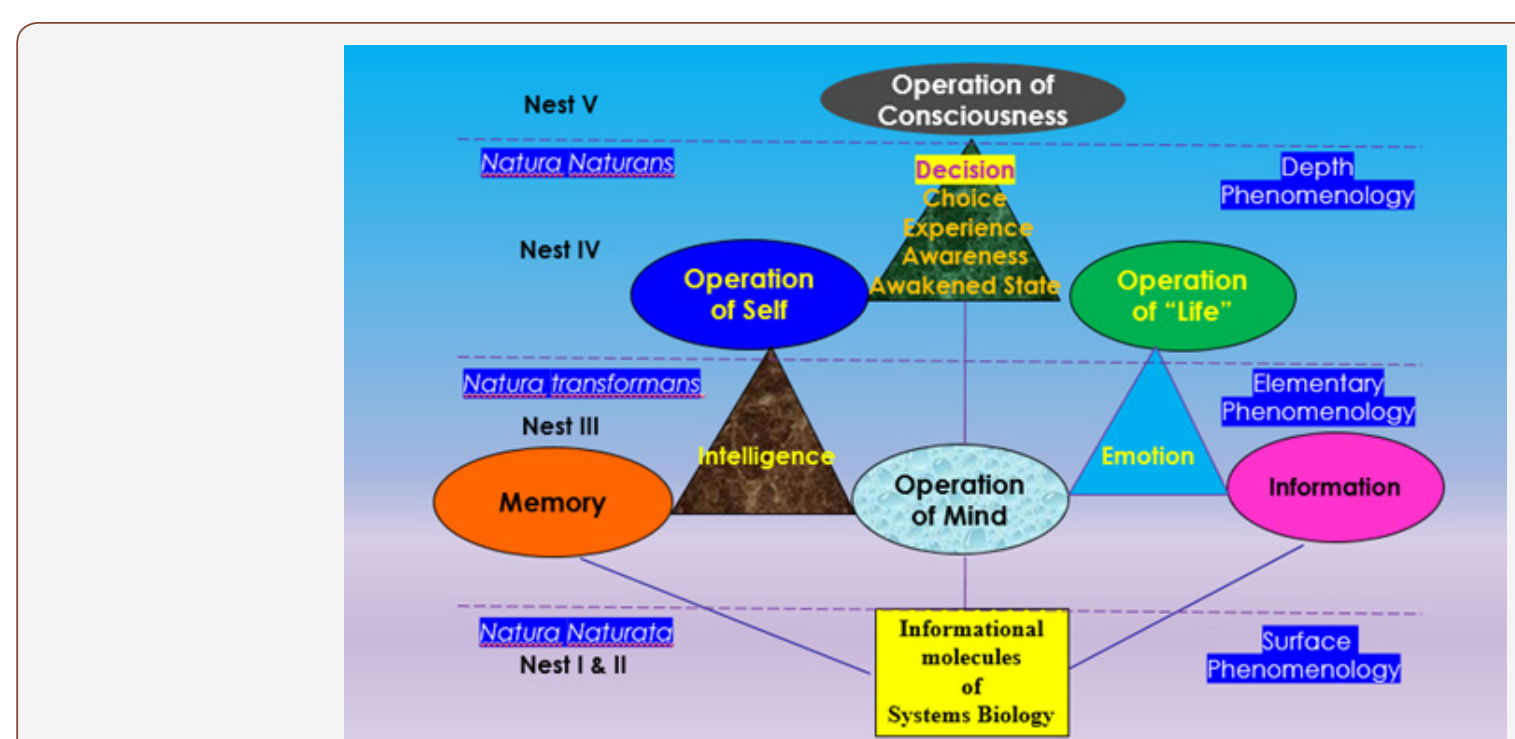

Figure 4: Stratified and hierarchically nested decision-making labyrinth of the Cognitive Orchestra. The members of the orchestra, their operation and their tentative location in phases have been shown. Subdivisions of nature's nest have been outlined. Phenomenology has been shown to be sratified in layers of systems science. The pathway is not straight but appears labyrinthine.

In this orchestra, consciousness for most of the occasions remains a visitor in the gallery, often is itself a sportsman in the field, always the leader (captain) of the team, occasionally intervenes as referee of the game, perpetually remains a creative organizer and at the depth itself is the ground. Compare these roles as published in our earlier paper, The cognitive canvas [20], as supporting consciousness, participating consciousness, intervening consciousness, creative consciousness and the ground consciousness. As evident from the model of this orchestra, none of consciousness, self, life and information has any direct connection with the material plane. It is through the mind, all consciousness, self, life and information connect with the matter. Mind is the final common pathway for material realization of the collective communication of the psychic orchestra as signal. That is why mind is so precious and significant organ of any living entity. Consciousness in human behavior manifests through mind. Mind is essential for love and compassion to manifest in the real world. Mind is the only vehicle for consciousness, life, self and information to reach the material world! This explains why the major doctrines of Buddhism works for health and hygiene of this organ, Mind. The communication pathway, however, is labyrinthine. The Light source which guides the direction of the travel through this labyrinthine path is consciousness.
Another interesting feature in this orchestra is the role of information, the operation of which is limited only in the immediate sub-quantum plane, along with that of mind and memory. Without any contribution from mind and information, one can remain awake being aware of their non-contribution in such mindless awareness. Mindful awareness accommodates mind and its contents. The orchestra is literally a juggernaut; it is overarching and huge, powerful and dynamic (in India, this might be compared with Lord Jagannath acting over our head), which acts as a system with several subsystems of operations and operators arranged hierarchically, although, in a labyrinthine manner. As subsystems influence the system, the system in turn determines through its subsystems the final outcome of the system.

Many advanced thinkers in Physics have worked for physics of "life" (e.g., Erwin Schrodinger, Paul Davis, Christian de Duve) [21-24], physics of "self" (e.g., Fred Alan Wolf, Amit Goswami) [2526], physics of "mind" (e.g., Stapp, Meijer and Korf, Rein) [27-29], physics of information (e.g., Gerad 't Hooft, Brukner and Zeilinger, Jude Curivan, Adrian Klein) [30-33]. However, there is no published literature, which speaks for interrelatedness of life, self, mind, information and their collective contribution for the holonomy of the Whole in the context of science of consciousness. At this 
point (space) and moment (time) of vacuum, the model of the orchestra stands erect and high as the inter-phase between brainindependent and brain-confined consciousness.

\section{The Inter-Phase}

According to the view expressed in this paper, consciousness is one. It is singular! Consciousness is both inside and outside the brain with respective contents. It is the brain, which divides consciousness into two, and creates the sense of multiplicity of consciousness. Understanding of the orchestrated operation carried out in this inter-phase helps in explaining the singularity and multiplicity of consciousness in appropriate context. This interphasing is the focus of the Supracortical Worldview. In the context of neuroscience, supracortical consciousness appears inexhaustible within cortical limits. It continues to work by bridging only one divide, the chasm between brain-bound and brain-independent consciousness. The idea transforms other existing divides such as divide between right brain and left brain, emotional and thinking brain, male brain and female brain.
In the context of social science Supracortical Worldview works for bridging also one divide, the chasm between brain-bound conscience of the individual and brain-independent collective conscience of the society, the chasm between individual conscience and collective conscience irrespective of race, cast, creed, religion, political affiliation and culture of the subject, irrespective of his/ her genetic and memetic background, irrespective of age, gender and evolutionary status of the subject. This "grand canyon" in supracortical Worldview is the cause of creation of all such divides within the brain and in the society and culture. To put it simply, this canyon is the divide between brain-bound consciousness and brainindependent consciousness. On one side, there is consciousness trapped within the brain, which could be described as "poor" in terms of economy and the "victim" in terminology of social justice. However, the imprisoned "self" in the brain has been ever-aspiring to become free and to come out of its enslavement and victimhood in order to build up connection with the inexhaustible boundless brain-independent consciousness especially during economic downturn of the brain (Figure 5)!

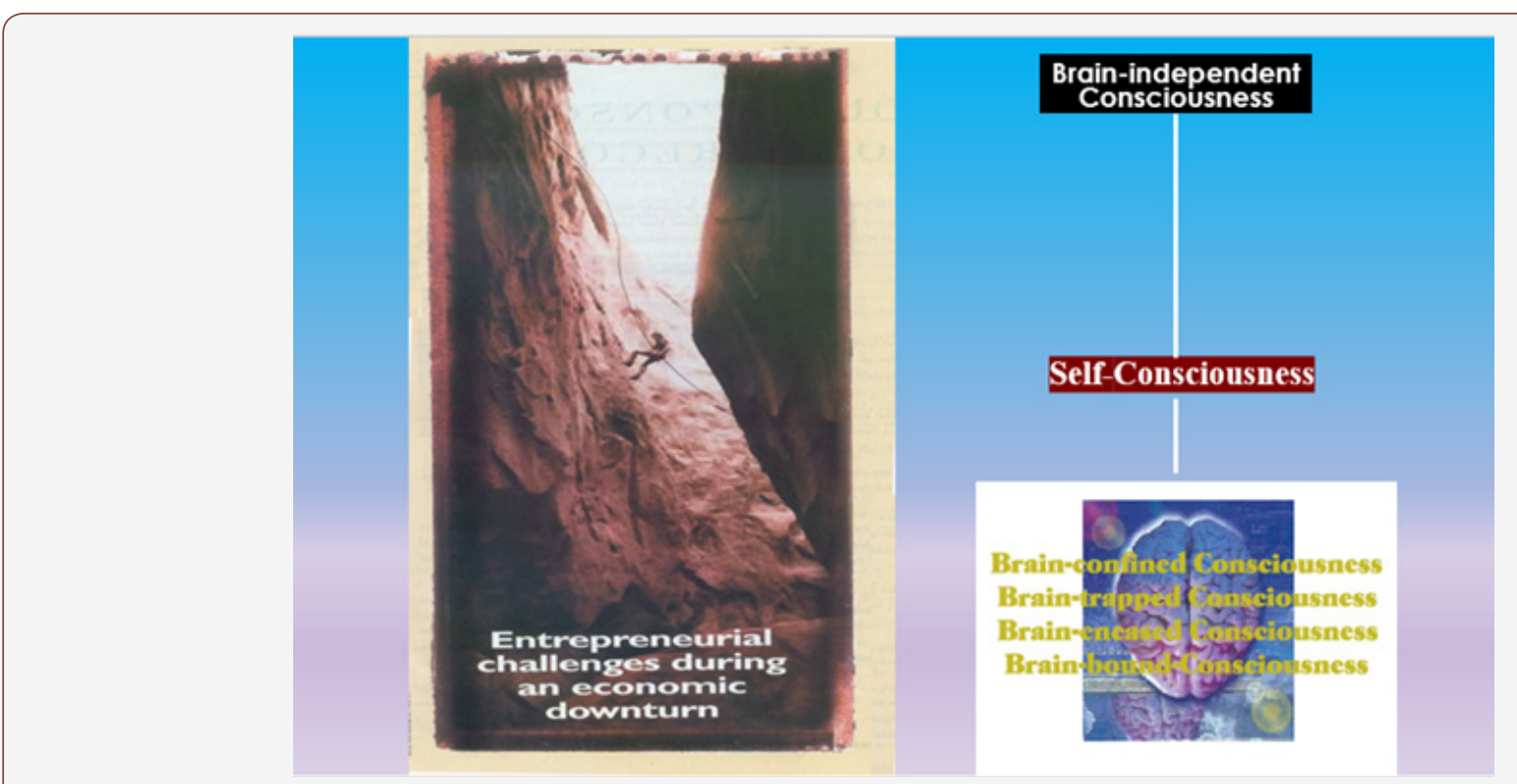

Figure 5: Consciousness within the brain could be called brain-trapped, brain-confined, brain-encased or brain-bound consciousness. Liberation of this economically "poor" and "imprisoned" consciousness and connecting it with ever-free boundless brain-independent consciousness is primarily a function of self, an important member of the orchestra.

Here is the operational worthiness of an inter-phase [34], which is orchestrated with its operators and operations. The orchestrated operations as described above (Figure 4) in the sub-quantum and sub-sub-quantum phases of nature operate as inter-phase between brain-bound and brain-independent consciousness, as a governing bridge between the finite brain and an endless Infinity! This inter-phasing is bidirectional. The members of the orchestra and their operations work for what in philosophy has been called naturalization of the transcendental and transcendentalizing of nature.

This inter-phase has an essential role in all and every experience whether this is of sensory, non-sensory or extrasensory in origin. Inter-phasing is probably not necessary as long we are obsessed with percept in the senses or concept in mind or even with complex knowledge owned by self. Inter-phase is essential when one is going for any experience. During an experience, inside becomes an outside reality. Profundity of the experience increases the objectivity of such reality.

\section{Consequences of the Proposition}

From the propositions above on the cascade of steps in communication, structure and operation of the cognitive orchestra and the inter-phase between brain-bound and brain-independent consciousness, one could look into five following areas.

A. Working definition of consciousness

B. Quantum-classical transition

C. The layers between neural signaling and consciousness 
D. Dark Energy and Zero-Point Energy (ZPE) State of the Brain

E. New way of classifying Psychiatric and Psychological illness

\section{A. Working Definitions of Consciousness}

From the orchestra and the inter-phase we are led to at least five working definitions of consciousness.

1. Consciousness is which looks after what all are happening in mind, self and life in terms of information management. This definition of consciousness is from ontological point of view.

Consciousness here is the MD (Managing Director) and the proposed orchestra constitutes systems for managing information. If information is discounted from the scenario, there is no need of consciousness! With this definition of consciousness, the discipline of self-management has been brought under systems science.

2. Consciousness is becoming aware of awareness, thinking about thinking, feeling about feelings, sensing of the senses, intuiting about intuition. Consciousness is the "I" taking care of me and mine. This definition of consciousness is from epistemological point of view and is studded with endless recursive processes. Consciousness is the chief of an orchestra constituted by powerful autonomous causal members each having infinite recursive property. Consciousness is the Information about information. Consciousness is the Mind of all minds. Consciousness is the Life of all lives. Consciousness is the Self of all selves. From this definition, we derive the systems psyche [35], and successfully brings consciousness within systems science, perhaps fulfilling an unfulfilled dream of Carl Gustav Jung and helping the attempt by Krippner and Comb [36].

3. Consciousness is Conscience from which generate Aesthetics and Ethics, which support Values upholding the sensible and visible Laws and Rules. This is a working definition of consciousness from axiological point of view. Consciousness is the judge. Finer discrimination is done by conscience. The orchestra forms the judicial system (Figure 6), where the values are upheld (by Mind) on the prescribed rules (as observable by Senses) with aesthetics (supported by Life) and ethics (supported by Self), united at the top by conscience. The logical brain, predominately the masculine brain, the left cerebral hemisphere processes information for formulation of ethics. The emotional brain, predominately the feminine brain, the right cerebral hemisphere processes information for expression of the senses of Aesthetics. Conscience unifies the right and left divide at the top and helps values at the bottom to support the laws and rules. Attitude is an outcome of unification of horizontal (left and right brain) and vertical divides (cf., Triune brain) of the brain. Attitude is an integrated combined output of conscience, ethics, aesthetics and values.

There are volumes of literature on each of rules and laws, on values, aesthetics, ethics, conscience and attitude individually. No single paper could be found on their comprehensive relationship. Since all are interrelated, and no comprehensive relation amongst them is available, no comprehensive definition could be found for any of them. At this point (space) and moment (time) of vacuum in the literature, this axiological definition of consciousness defines attitude, which is customarily said to be an output of affective brain. At this defining moment of comprehensive interrelatedness, the proposed model (Figure 6) opens up the door for (i) systems science for AETCOM (Attitude, Ethics and Communication) module in education (ii) value-education in social science (iii) soft skill learning in community medicine and public health and, (iv) the discipline of Law and Judiciary. Absorbing the vacuum, the model stands erect to show how law makers make rules on the basis of contemporary values in the mind of the society based on community's ethics and aesthetics followed in the human lives, supported overarchingly by collective conscience of the nation. Rules and laws are therefore changeable according to the need of the society, community and the nation. Constitution of a nation is also amended as and when it is necessary.

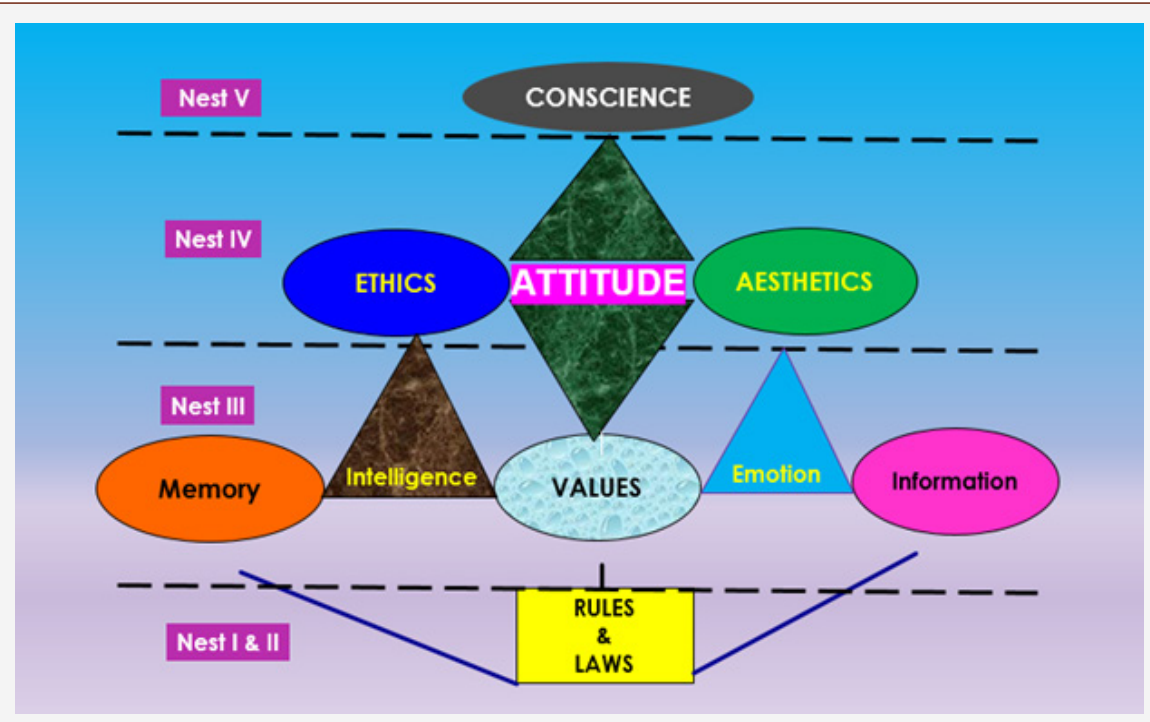

Figure 6 : From Conscience generate Aesthetics and Ethics, which support Values that uphold the sensible and visible Laws and Rules. Attitude is an outcome of interaction of conscience, ethics, aesthetics and values. The discipline of Law and Judiciary has been shown as a part of systems science with this law-making cascade. 
No rule or law is foolproof! There are loopholes. A man with doubtful integrity is he who knows the rules, knows the loopholes of the rules as well. However, he uses the rules to trouble others. He utilizes the loopholes of the rules for self-proliferation. In fact, this is a property of a virus, which knows the rules of the cell better than the cell itself. It also knows the gaps in the cellular rules, which are fully utilized for its own proliferation using cellular DNA. A diplomat also knows the both sides of the rules and uses them on negotiating table. The judge in a court knows both sides of the rules and assesses the gravity of breakdown of rules using both the angles.

The interrelationship described helps the judicial system to go into the depth, reading in between the lines of a law book, while framing the judgment on a complex case to uphold the fabrics of the values prevailing in the society. In the rarest of rare occasions, where crime is being conducted not merely by breaking the rules but destroying the values, defacing the ethics, aesthetics and the collective conscience of the society, there prevails the scope for capital punishment (Figure 6)!

4. Consciousness is phenomenal. Consciousness is the phenomenon. Consciousness is the center of all phenomenology in nature; surface phenomenology (at classical and quantum nests of nature), elementary phenomenology (of mind, information, memory and intelligence at sub-quantum nest of nature) and depth phenomenology (of self and life in sub-sub-quantum nature). Consciousness creates phenomena which eventually become sensible as organized events. Consciousness regulates, modulates, changes and manages phenomena. All phenomena are absolved by consciousness, absolved in consciousness. This is a definition of consciousness from phenomenological point of view. With this definition, phenomenology could be brought within systems science.

The self, which on behalf of consciousness operates as the chief executive officer (CEO) within the systems is at the center of phenomenology for the systems. As mind is sensitive to information, life is sensitive to imbalance in homeostasis, so the self is sensitive to phenomena.
With this definition of consciousness, we open up a new frontier in cell biology, that is to study systems cell with phenomenological approach. The constituents of surface phenomenology within the cell are evidenced in signal networking, signal transduction and messaging through information highway of microtubules, for example. The elementary phenomena, as mentioned in author's paper on systems psyche [35], are love, sex, ego (conditioned existence), life (birth) and death. Such phenomena are observed as cell division (birth, new life), apoptosis (death), restricting or killing of microbes by macrophage or NK (natural killer) cell (an example of ego, conditioned property), fertilization of ovum by spermatozoon (sex), and voluntary transportation of ATPs from astrocyte to neuron without asking for any favor in return (an example of love). We are not aware of any specific or distributed position of operators and operations of "psyche" such as self, life and consciousness within the systems cell. Had it been known, one could easily look into the depth phenomenology within the systems cell. Breaking open this juggernaut could be an alternative approach to demystify the deep mystery of cellular origin and mechanism of disease.

Consciousness, from its ontological authority, axiological strength, epistemic expertise and phenomenological dexterity brings certitude in the world of uncertainty, brings circumstances beyond control under control, brings order following chaos and catastrophe.

5. Consciousness is the repository of all kinds of memory, which is holographically encapsulated within the system, as well as independent of the systems (as wisdom). The overarching memory liberates within the cognitive systems as experiential memory, may be in several layers, that is represented within self as episodic/ representational memory and within mind as semantic memory.

Memory even in holographic storage form is, therefore, hierarchically organized, some of which disturbing a patient are explored as "malware" by deep hypnotic procedures. Memory loss in dementia is also stratified. Helping regaining some of such "good-ware" memories, therefore, requires a multi-stair guide to the patient by physician.

\section{B. Quantum-Classical Transition}

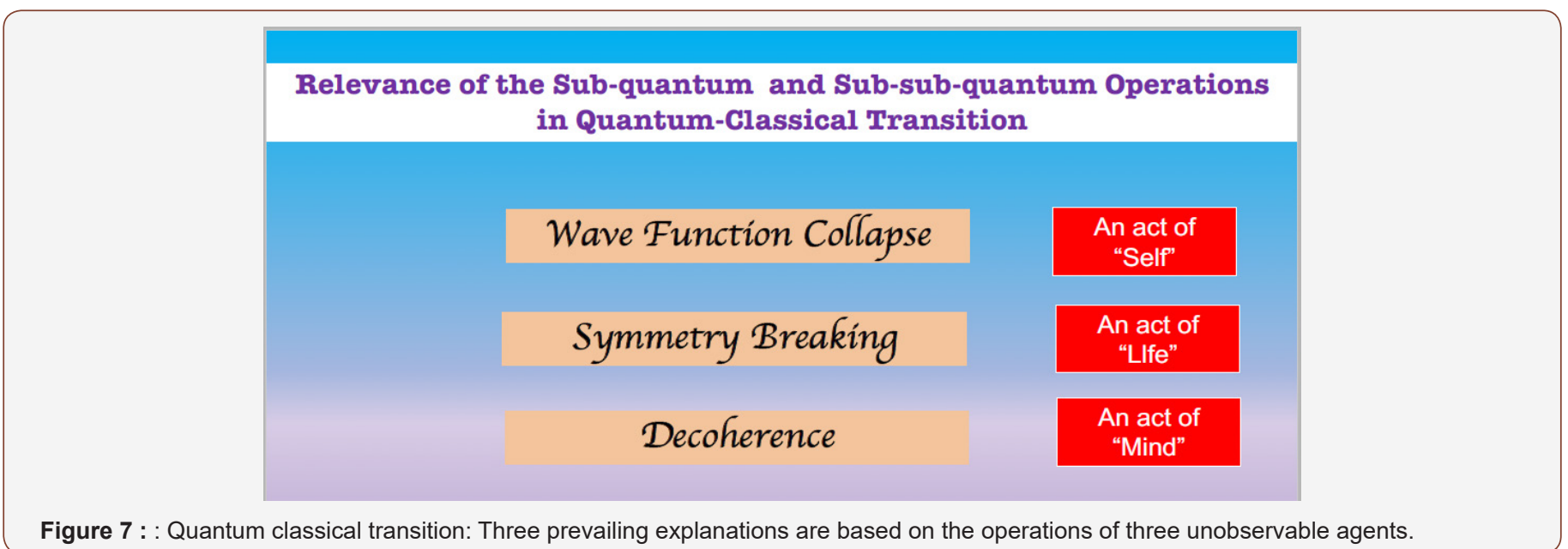


We still do not know the mechanism of quantum-classical transition in physical phenomenology. There is relevance of the operations described above in sub-quantum and sub-subquantum phase of nature for manipulation of quantum events and their transition as classical observable events (Figure 7). An act primarily of Self's initiative on quantum events could lead to wave function collapse. An act primarily of 'life's initiative could describe this quantum-classical transition as symmetry-breaking process. An act primarily of mind's initiative describes this transition as decoherence. So far, we know only these three explanations on mechanism of quantum-classical transition (Figure 7).

\section{The layers between Consciousness and Neural} signaling

The scheme we are going to describe here is relevant in neuroscience since it points out and generates approach for investigation of the layers between neuronal signaling and conscious experience and even consciousness. At present, the prevalent opinion is that specific spatiotemporal pattern of signaling is responsible for generation of conscious experience. The development in science what has taken place over last two to three decades in this direction is from classical electrophysiology of synaptic network (objectified by EEG pattern, Brain mapping etc.) to quantum level cell signaling in neural network and then to the theoretical computational neuroscience [37] regarding encoding, decoding and recoding of bits. Up to this level of development, everything is sensible, measurable and reducible in our objective, positivistic and reductionist approach in scientific enquiry. What happens next at deeper level is not known.

Here comes the importance of inter-phasing system, which operates in the sub-quantum and sub-sub-quantum depth of nature to connect the domain of senses with the domain of consciousness. In this phase, we have proposed the existence and operations of non-observable influential(s) which are autonomous potent causal agents. In the sub-quantum domain, there are operations of mind and information, emotion and intelligence, in the sub-sub-quantum domain are the operations of self and life, which are in connection with the operation of consciousness (nest V of nature in Figure 4).

Let us describe this cascading down along the ladder of cognition [38] with the help of Figures 3 and 8 (Figure 8).

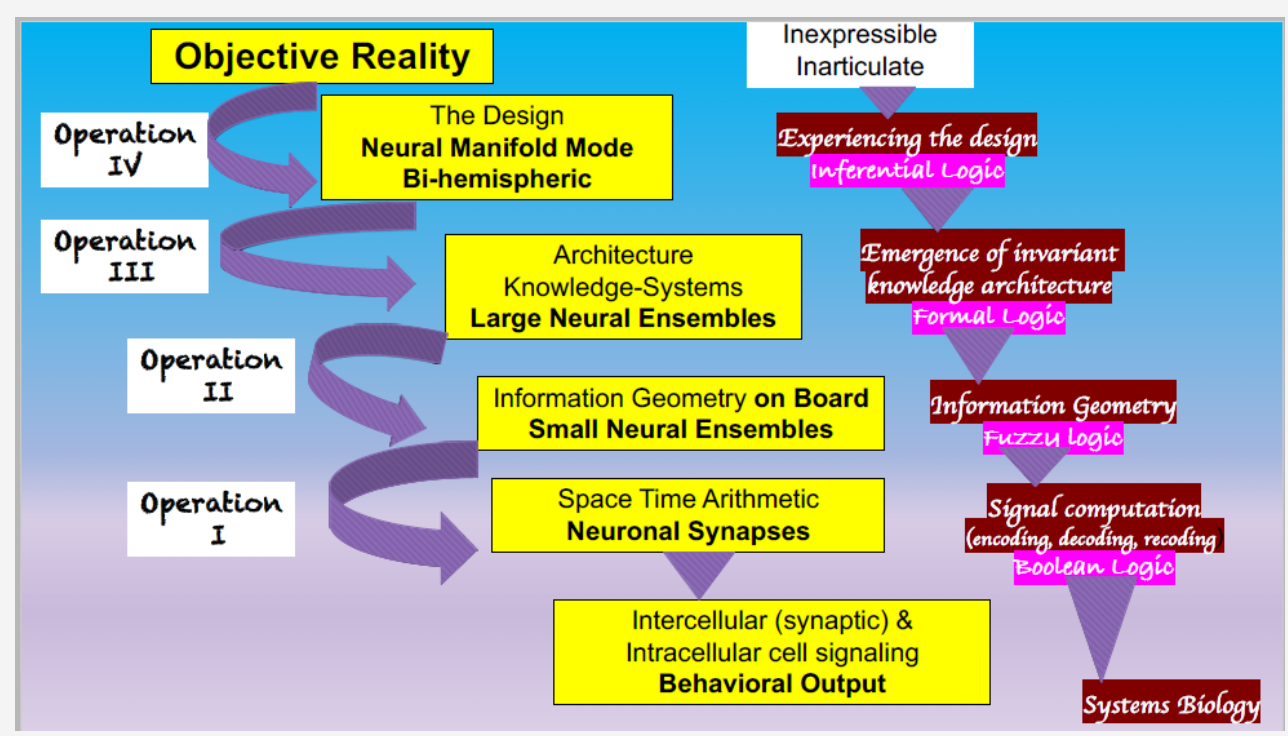

Figure 8 : The layers between consciousness at the top and neuronal signals at the bottom. Cascading operations are on the left and the cascading transition of logic are on the right side of the figure. Successive involvement of neural manifolds, large scale neural assembly, small scale neural assembly and synapses are shown in the yellow boxes, which also shows succession from OR to Design, Architecture, Information geometry, Arithmetic to behavioral output.

Consciousness to conscious experience is the first step. The OR, apparently inexpressible and inarticulate, condensed in nature as crystal information represented as the point of wisdom or the source of Worldview cascades down as an experience of an overarching design of the quality, represented by information manifold resulted from exalted decomposition of the crystal information, supported within the brain bi-hemispherically by neural manifold. This is the topmost operation, designated as Operation IV, executed primarily by consciousness. Operation of consciousness is the first operation to ground quality into quantity, from a point to piles of spheres, represented as information manifold.

By Operation III, executed primarily by "life", information manifold is cascaded down to 'information of the whole', Gödelian information, (recognized as the crux of a theory), represented in the invariant symmetry of knowledge systems supported within the brain by large neural ensembles where inferential logic is cascaded down to formal logic.

By the next operation, Operation II, executed primarily by self, the phenomenon gets down from symmetry to geometry of relevant pieces of information on board, expressed, in essence, as a hypothesis supported within the brain by small neural ensembles where information operates following a fuzzy logic.

The last operation in this downward causation, which is the first operation in bottom-up approach, Operation I, entails conversion of geometry of semantic information, internally felt as a concept, 
into arithmetical expression of energy in terms of its occupation of specific space per unit time, i.e., as signal frequency of the sensory apparatus, which are computed (encoded, decoded, recoded) by Boolean logic supported neurologically by synaptic activities, which is felt as perception (Figure 9).
Consequent to intercellular signaling through cell to cell junction or synapses follows intracellular signaling that lead to behavioral outcome working through apparatus of genomic, proteomic and metabolomic of the concerned cell. This is the proposed way how the systems psyche connects consciousness with the systems biology!

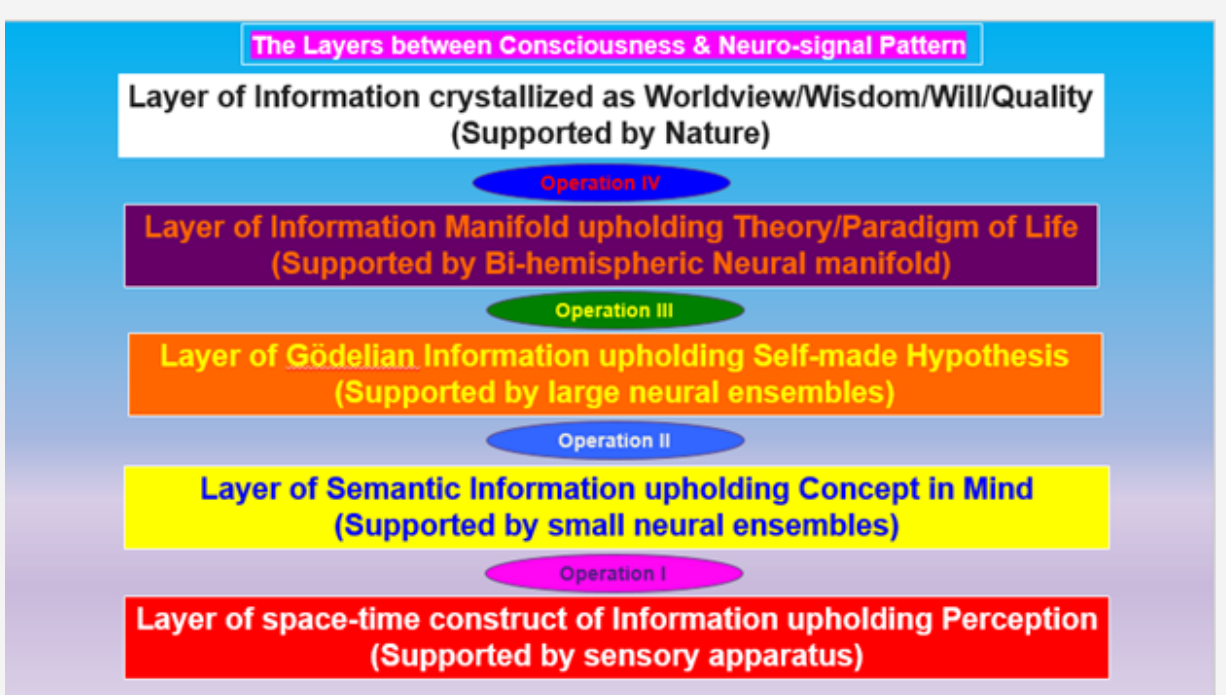

Figure 9: The layers between consciousness and conscious experience on the top and space time world of sensory perception at the bottom.

From bottom (Figure 9) up, there are layers of space-time construct of information, the layer of semantic information, the layer of Gödelian information, the layer of information manifold and the topmost layer is the layer where the quality, the Worldview or the wisdom, crystalized as information crystal.

\section{Dark Energy and Zero-Point Energy State of the Brain:} The Relevance of the Orchestra

A live entity is characterized by its ability for homeostasis; uncertainty-certainty homeostasis, symmetry (breaking and making) homeostasis and dark energy-visible energy homeostasis. The brain is a live community of hundred billion neurons and two to ten times of glial cells. Therefore, the brain participates in all three homeostasis mentioned above.
The brain transacts with dark energy during its cognitive gymnastics [39]. While going up the ladder of cognition, the visible energy is converted into dark energy which helps in structuration of information into knowledge, knowledge into experience and experience into wisdom. While descending down the ladder, the dark energy is converted into visible energy within the brain. In sub-Planckian scale, even dark energy could be converted into dark matter. Conversion of energy into matter is not observed to happen in nature of Planckian scale, probably with one exception of formation of Higgs Boson (popularly known as God particle) that too probably has transaction with sub-Planckian scale of nature. The zero-point energy state is where the domain of dark energy and visible energy meet (Figure 10).

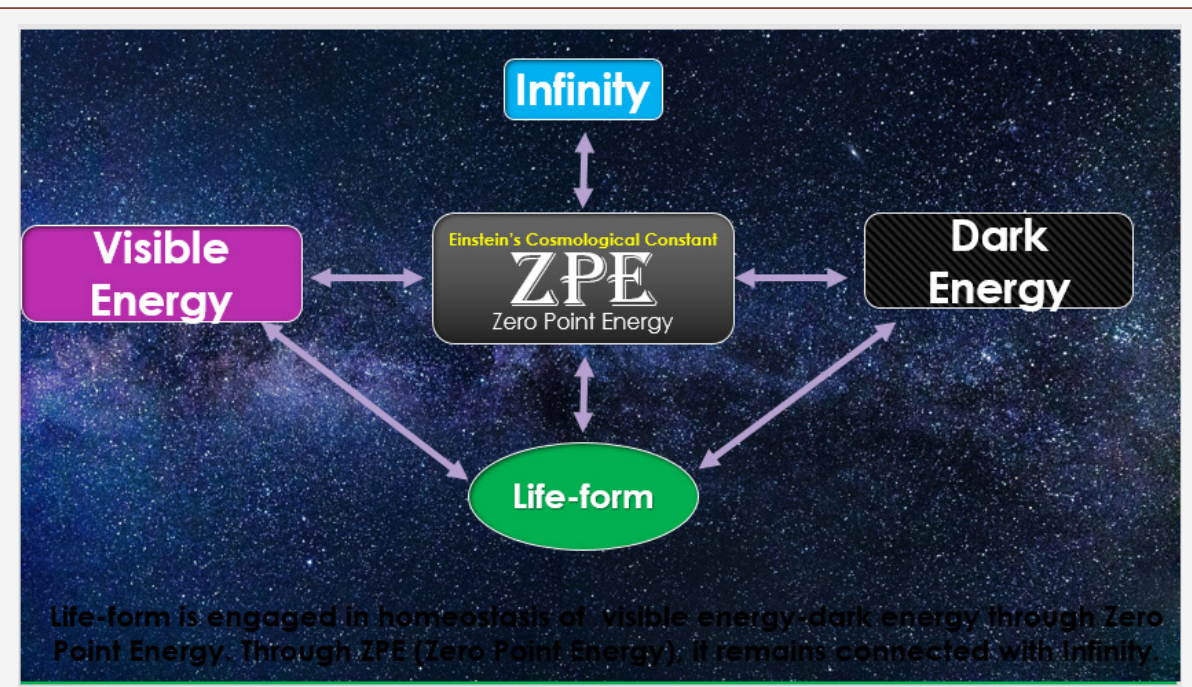

Figure 10: The domains of visible energy and dark energy meet at zero-point energy state. Through ZPE state a living entity and the brain of a complex organism communicate with the Infinity. 
This meeting ground of dark energy and visible energy is also the site of operations of the cognitive orchestra, which is possessed by all live entities, life-forms at various stage of evolution. Zero is read "zero" for matter, but not for information, life, mind, self and consciousness. Operations of material world and operations of psychic world join here. Through ZPE point only, the finite communicates with the Infinite. Information holograph shapes into space time world [40]. The cerebral cortex communicates with supracortical domain. All dimensions (space, time) originate from this ZPE. At the ZPE point, all dimensions, lower and higher, vanish.

Zero-point-energy state of the brain is a state or phase of the brain when the brain as a whole, at its classical-, quantum- and phenomenological integrated state, remains conducive for being nourished by the Infinity through the orchestra described in this paper. Regarding the dark energy-visible energy homeostasis inside the brain, the state is at its optimal equilibrium, where quantum fields serve as the messenger of Infinity. Fred Wolf observes the mind of God in these quantum fields [41]. More the brain remains in this most comfortable and restful state more is the chance of the anabolic processes of healing, repair and regeneration within the brain to be at their peak. There is possibility of huge information gain by the brain at this state. At the ZPE state of the brain, the brain reverts back to its regenerative best. The door of a new brain formation opens up (Figure 1).

The "brain" of a brain might be an imagination of an artist as shown in the Figure 11, but is proposed to be a reality with supracortical worldview and downward causation (Figure 11).

Chapter 8

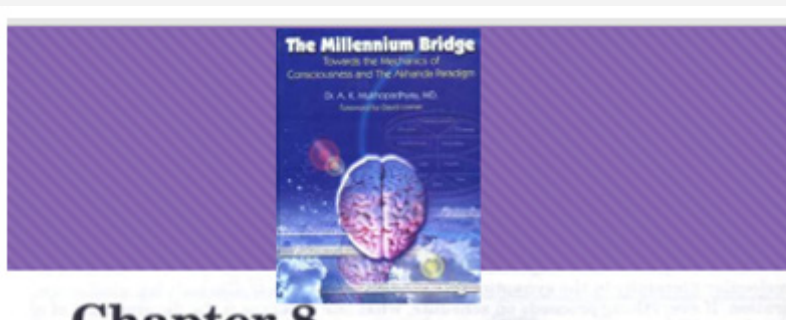

The Brain of a Brain

The Sine-qua-non of the New human species emerging on this Earth

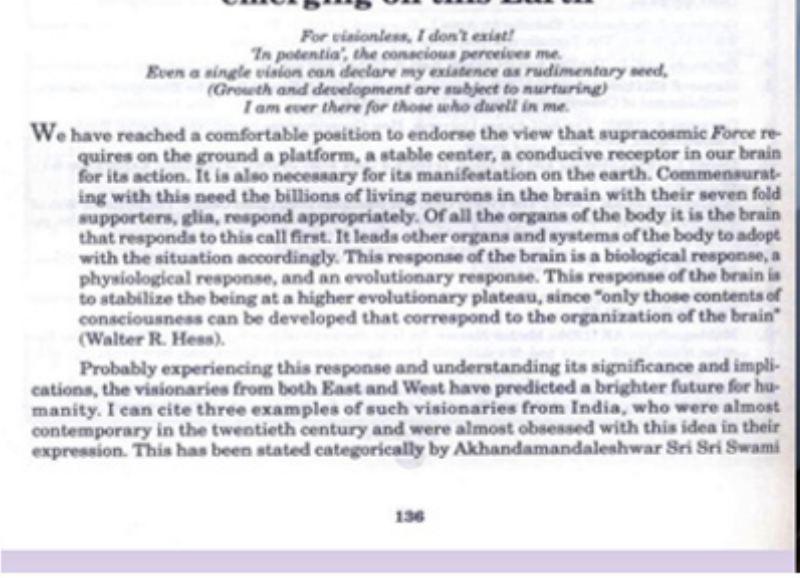

Figure 11: A photograph of a sculpture showing heads growing over heads.

The "Brain" of a brain is the sine-qua-non for a new species emerging on this earth. The orchestra described in this paper is also significant for the matter world since it could possibly help to chalk out a pathway from dark matter to dark energy to visible energy and vice versa. We have at his stage of development of science no equipment, instrument or device which could convert dark energy into visible energy except mind. Inventing a technology which could perform this mind-like operation could help us in harnessing dark energy, which might resolve the energy crisis of the world.

The picture of the universe we draw and work with is our cognitive (cortical) construct! As soon as we look through a "window" in the cortex, we are at the plane of the largest intellectually comprehensible systems of multiple universe(s), The
Multiversity. At this depth, exists the Source-field of inexhaustible "life". The Multiversity and Supracortical Consciousness, therefore, seem to be twin Worldviews, one relevant for astrophysics and the other relevant for neuroscience. When a life-form, a living cell becomes aware of this eternal life in the sub-sub-Planckian nature, it seems to be situated at the creative fountainhead!

\section{E. New way of classifying Psychological and Psychiatric} Diseases

Based upon the propositions in this paper we are now in a position to have a new look into classification of the psychological and psychiatric illness. The inter-phasing orchestra between the transpersonal and personal plane have potent causal agents such as consciousness, self, life, mind and information. The psychosomatic 
connection is executed by mind's operation, that converts information into signal and signal into information. Disease could originate from disturbance in one, two or more or all of the causal agents regarding their autonomy, operation, interconnectedness in their solo, joint or combined output in behavior. In the systems psyche paper [35], the possible molecular links have also been suggested. It seems that this way of classification would be a coherent component of systems science as a whole, could be easily translatable in clinical practice both for diagnosis and management of patients. However, at this stage, this is one man's contribution. The Neurological, Psychological and Psychiatric societies all over the world might take note of this for deliberation on this issue.

\section{Concluding Remarks and Perspectives}

The proposed model connects the highest grade of quality with the bottom of objective, positivistic and reductionist science in a systematic way. This shows a way of cracking the reverse "Hard Problem" of consciousness. With the emergence of this bidirectional pathway effective both in top down and bottom up approach, the model is sure to help expansion of the horizon of science. The models and the postulates derived from the deconstruction and new construction merit further investigation. My colleagues find really a huge scope for several multidisciplinary, interdisciplinary and trans-disciplinary projects. My guess is that it will not leave any discipline untouched! On the contrary, this is likely to accommodate all relevant disciplines to build up systems science with profound impact on humanity, on its memes, genes and environment. The Global Consciousness Project (GCP) initiated by Roger D. Nelson [42] in 1998 and funded by Institute of Noetic Science to detect possible interactions between "global consciousness" and physical systems such as random number generator is likely to consolidate further. The functions and the purpose of having a brain as a precious gift from nature, are likely to be evaluated contextually with new-found relevance in its cognitive evolution, which could be supported by evidence at molecular level in genomic, proteomic and metabolomic. Regenerative medicine with new healing methods and technology (Information holograph, harnessing ZPE State) are likely to take a new shape within the supracortical Worldview. The puzzles and the paradoxes of quantum physics would be seen as creation of information! Life would be considered more precious in science, in humanity and for the spirit. In medicine, this scheme has translatable effect on regenerative medicine, psychosomatic medicine, longevity medicine and personalized medicine. Neurology, Psychology and Psychiatry are to be first benefitted from the propositions in this paper. In technology, this scheme have definite impact on new healing methodology (harnessing ZPE state, information holography, and neutrino beaming), inventing device that could harness dark energy for daily common use, and in scaling a greater height in the field of Artificial Intelligence and Artificial Life.

\section{Acknowledgement}

The author presented this paper at Dayalbag Educational Institute at Agra, India on 18th of May 2019, as a plenary speaker on the occasion of DEI-TSC-2019, and DEI-40 years. The author expresses his gratitude towards the Chairman, President and Director of the Institute and the organizing secretary of the functions.

\section{Declaration}

There is no conflict of interest with anyone in publication of this article. No funding agency has any financial contribution in this publication.

\section{References}

1. Barrs BJ (2017) The Global Workspace Theory of Consciousness. In: Susan Schneider, Max Velmans (Eds). Willey Online Library Book.

2. Tononi G, Boly M, Massimini M, Koch C (2016) Integrated information theory: from consciousness to its physical substrate. Nature Reviews Neuroscience 17: 450-461.

3. Block N (2007) Consciousness, Function, and Representation: Collected Papers Bradford.

4. Hameroff S, Penrose R (2013) Consciousness in the universe. A review of the 'Orch OR' theory. Phys Life Rev 11(1): 39-78.

5. Meijer DK, Raggett S (2014) Quantum Physics in Consciousness Studies.

6. Beichler JE (2017) Physics of Consciousness: The path to understanding consciousness.

7. Mukhopadhyay AK (2014) From Quantum to Consciousness: A long way to go! Chapter 22 in, Brain Mind Cosmos: The nature of our existence and the Cosmos. Ed. Deepak Chopra. Pub. Deepak Chopra.

8. Gray CB (2007) Consciousness Studies: The Emerging MilitaryIndustrial-Spiritual-Scientific Complex. Anthropology of Consciousness 18(1): 3-19.

9. Panidi, K (2017) Nobel Prize in Economics - 2017: Richard Thaler's Contribution. HSE Economic Journal, National Research University Higher School of Economics 21(4): 702-720.

10. Mukhopadhyay AK (1985) States of Consciousness a holistic hypothesis. Supracortical Consciousness an existing Reality, In, Frontiers of Research for Human Biologists Next Hundred Years. Conscious Publications, New Delhi, India, pp. 1-6.

11. Mukhopadhyay AK. http:// www.akmukhopadhyayconsciousness.com

12. Mukhopadhyay AK (2000) The Brain of a Brain. The sine-qua-non of the new species emerging on this earth. In The Millennium Bridge. Conscious Publications, New Delhi, USA, pp. 136-152.

13. Moore MAS (1999) Turning Brain into Blood Clinical implication in Stem Cell research in Neurobiology and Haematology. N Eng J of Med 341(8): 605-607.

14. Kim J (2000) Causation and Mental Causation. In, Contemporary Debates in Philosophy of Mind. Eds. Brian P. Maclaughlin and J D Cohen Blackwell Publishing. 227-242.

15. Kafatos M, Tanzi RE, Chopra D (2011) How Consciousness Becomes the Physical Universe. Journal of Cosmology 14: 1-11

16. Pribram K H (2011) Brain and perception: holonomy and structure in figural processing. Routledge, New York, USA.

17. Libet B (2006) Reflections on the interaction of the mind and brain. Prog Neurobiol 78(3-5): 322-326.

18. Kak S (2009) The Universe, Quantum Physics and Consciousness. J Cosmol 3: 500-510.

19. Klimo J (2010) The Past, Present, and Future of Consciousness Studies. Paper presented in United States Psychotronics Association Conference.

20. Mukhopadhyay AK (2018) Cognitive Canvas: Molecular Embroidery, Fabric and the Base. EC Psychology and Psychiatry 7 (7): 428-439. 
21. Schrodinger E (1944) What is Life? Cambridge University Press, Cambridge.

22. Davies P (2001) The Origin of Life. When and Where Did It Begin? Science Reporter 84: 1-16.

23. Davies P (2001) The Origin of Life. How Did It Begin? Science Reporter 84: $17-29$.

24. de Duve C (2002) Life Evolving. Oxford University Press, Oxford, UK.

25. Wolf FA (1996) The Spiritual Universe. How Quantum Physics Proves the Existence of the Soul. Simon \& Schuster, New York, USA.

26. Goswami A (1993) The Self-aware Universe. How consciousness creates the material world. Tarcher/Putnam, New York, USA.

27. Stapp, HP (2012) Reply to a critic: Mind efforts, quantum zeno effect and environmental decoherence. Neuro Quantology 10: 601-605.

28. Meijer DKF, Korf J (2013) Quantum modeling of the mental state: the concept of a cyclic mental workspace. Syntropy Journal 1: 1-41.

29. Rein G (2017) Mind as a virtual phase-conjugated Hologram. Cosmos and History. The Journal of Natural and Social Philosophy 13(1): 1-13.

30. 't Hooft G (2005) Determinism beneath Quantum Mechanics. In, Quo Vadis Quantum Mechanics. In: A Elitzur, S Dolve, N Kolenda (Eds.), Springer, USA, pp. 99-111.

31. Brukner C, Zeilinger A (2005) Quantum Physics as Science of Information. In, Quo Vadis Quantum Mechanics? A. Elitzur, S. Dolev,N. Kolenda (Eds.), Springer: 47-61.
32. Currivan J (2017) The Cosmic Hologram. In-formation at the Center of Creation. Inner Traditions. Rochester, Vermount, Toronto, Canada.

33. Klein A (2019)(personal e-mail communication)

34. Mukhopadhyay A K (2016) Systems-bound and Systems-independent Consciousness Complex Inter-phase in between: From Narrative to Science. Psychol Behav Sci Int J 1(3): 555562.

35. Mukhopadhyay AK (2016) Systems Psyche: Its Structure, Operation and Possible Molecular Links. Psychol Behav Sci Int J 1(3): 555565.

36. Krippner S, Combs, AL 1998 A systems approach to creativity and consciousness. Systems research and behavioral science 15: 81-91.

37. Banerjee R, Chakrabarti BK (2008) Models of Brain and Mind. Physical Computational and Psychological Approaches. In: Rahul Banerjee, Bikash K Chakrabarti (Eds.), Progress in Brain Research168. Elsevier, Amsterdam, Netherlands.

38. Mukhopadhyay AK (2017) The Ladder of Cognition: Abstract Operations, Molecular Biology, Systems Science. Ann Psychiatry Ment Health 5(4): 1107.

39. Mukhopadhyay AK (2019) Zero-Point Energy State of the Brain. Archives in Neurology and Neuroscience 2(5): 2019.

40. Mukhopadhyay AK (2012) Information Holograph. The Structure, the Source and its Operation. International Journal of Bio Engineering Neuro Sciences and Technology 2(2): 12-32.

41. Wolf FA (2008) Is the Mind of God Found in Quantum Field Theory?

42. Global Consciousness Project. 